\title{
CENTRIFUGAL STRESSES IN ARBITRARILY LAMINATED, RECTANGULAR-ANISOTROPIC CIRCULAR DISCS
}

\author{
C. W. BERT School of Aerospace, Mechanical and Nuclear Engineering, University of \\ Oklahoma, Norman, Oklahoma
}

\begin{abstract}
The problem is formulated as one in the linear theory of thin, laminated, anisotropic elastic plates. A direct force-and-moment formulation is used, simplifying approximation is introduced and a closed-form solution is obtained. This solution exhibits bending-stretching coupling if the plate is asymmetrically laminated with respect to mass or stiffness or both. Numerical results typical of certain composite materials of current interest are presented. Specific laminates considered as examples include (1) glass-epoxy/steel, (2) cross-ply graphiteepoxy, and (3) various quasi-isotropic layups of organic fibre-epoxy.
\end{abstract}

\section{INTRODUCTION}

The problem of determining the elastic stresses in a thin isotropic circular disc rotating at constant speed about a normal axis passing through its centre (Fig. 1a) was attributed by Timoshenko (1)* to Maxwell in 1850. The analogous problem for a disc constructed of cylindrically orthotropic material was attributed by Lekhnitskii (2) to Glushkov in 1939. In such a material, the materialsymmetry axes are oriented along a cylindrical co-ordinate grid pattem (Fig. 1b). The technical manifestation of such a disc is exemplified by one filament wound so that all of the filaments are circumferential.

In certain products, such as grinding wheels, it is most economic from a production standpoint to construct the disc of rectangular orthotropic material. In this kind of material, the material-symmetry axes are oriented along a rectangular grid pattern (Fig. 1c). This rotating-disc problem was solved by Lekhnitskii in 1944 (3).

In some instances, it may be necessary to construct the disc by laminating it of multiple layers of different materials. For the case of an arbitrary lamination scheme of isotropic materials (see Fig. 1d for an example), this problem was solved recently by Bert (4).

The title problem may be considered to be a generalization of (3) and (4) and is solved in closed form in the present paper. It is exemplified by a disc laid up in a crossply arrangement of multiple layers of different materials that are rectangular orthotropic, such as material reinforced by glass cloth (5) or unidirectional filaments. Fig. $1 e$ shows a simple two-layer example of such a disc.

Another class of examples comprises the quasi-isotropic lamination scheme originated by Werren and Norris (6) and further studied by Tsai and Pagano (7) and by Bert (8). The application of this lamination scheme to rotating discs was patented recently by Rabenhorst $e t$ al. (9). Two simple examples of this lamination scheme are shown in Fig. 2.

The MS. of this paper was received at the Institution of Mechanical Engineers on 3rd May 1974 and accepted for publication on 27th January 1975. 3

* References are given in Appendix 3.

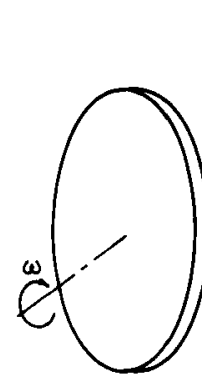

o

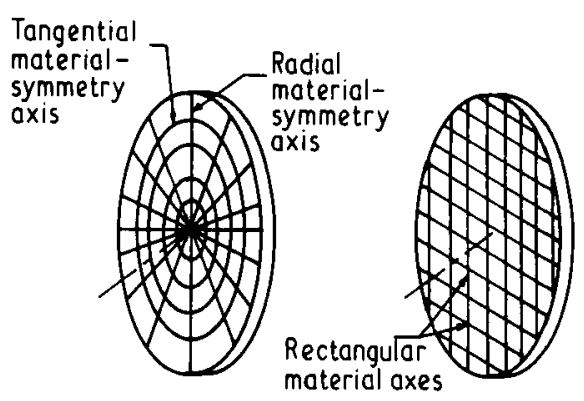

b

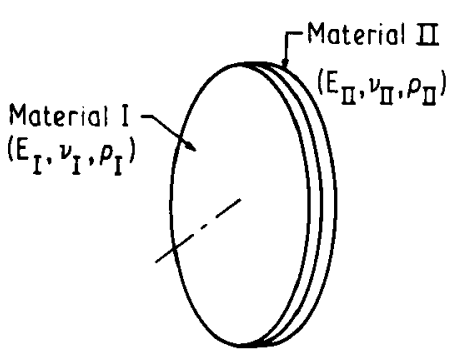

d

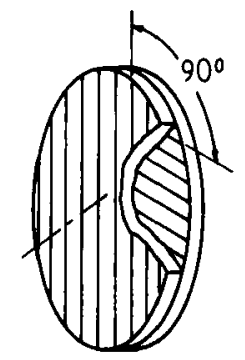

e $a$ Homogeneous isotropic. $b$ Single-layer cylindrically orthotropic. $c$ Single-layer rectangular orthotropic. $d$ Two-layer isotropic. $e$ Twocross-ply, rectangular or thotropic.

Fig. 1. Examples of circular disc configurations

It is to be emphasized that in lamination arrangements in which both the stiffness and mass are symmetrically disposed with respect to the midplane of the laminate, there is no difference between the functional form of the solution and the form for a homogeneous or single-layer disc within the context of thin-plate theory. However, if either the stiffness or the mass is located unsymmetrically with respect to the laminate midplane (or if both are), bending-stretching coupling takes place and changes the qualitative character of the solution. The bendingstretching coupling phenomenon has been demonstrated by 

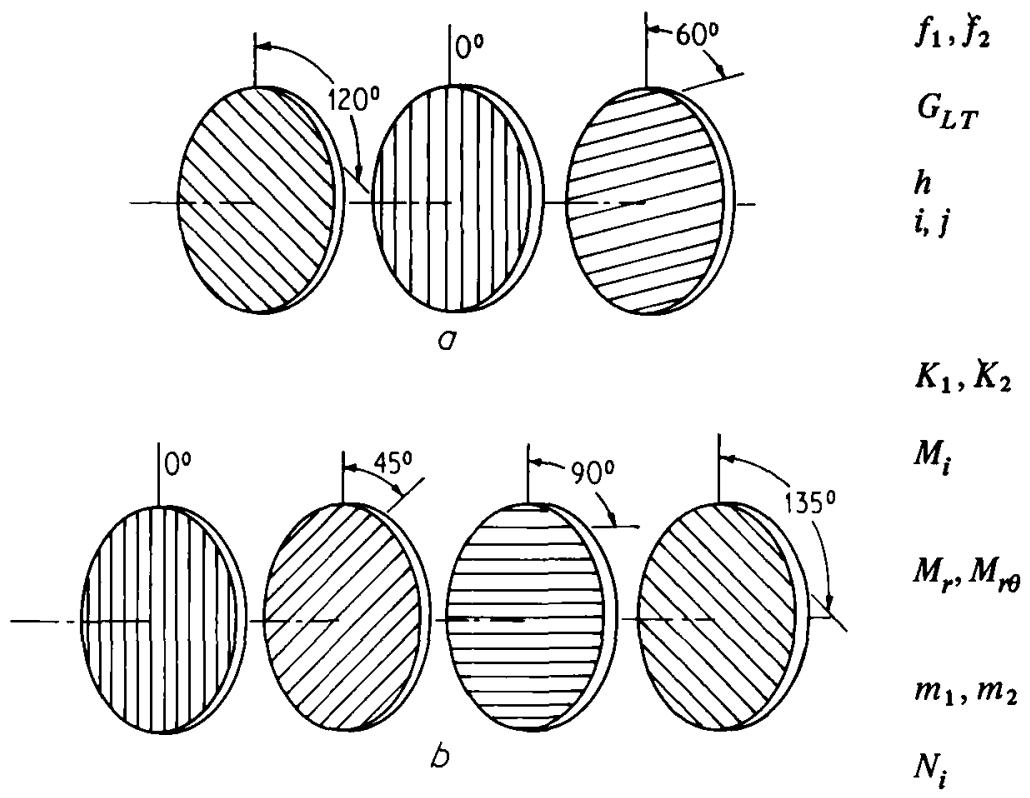

$a$ Three-ply. $b$ Four-ply.

Fig. 2. Exploded views of quasi-isotropic lamination schemes

many previous investigators in connection with normally loaded plates, see, for instance, Whitney (10) and chapter VI of Ashtons and Whitney's book (11).

The topic of laminated plates loaded by centrifugal action is not treated in the classical book on the subject (11). To the best of the author's knowledge, the only previous papers on the topic of rotating laminated plates are a plate-theory analysis by Deb (12), a classical-elasticity analysis by $\mathrm{Wu}$ and Ramsey (13) of a disc symmetrically laminated of isotropic material and Bert's previous thinplate analysis (4) of a disc laminated arbitrarily (either symmetrically or asymmetrically) of isotropic materials. The present solution, which employs thin-plate theory, is more general than previous analyses in that it takes into account both rectangular anisotropic material and arbitrary lamination symmetry.

\subsection{Notation}

$A, B, D, H$

$\left[A_{i j}\right],\left[B_{i j}\right],\left[D_{i j}\right]$

$\left[A_{i j}^{*}\right],\left[B_{i j}^{*}\right],\left[D_{i j}^{*}\right],\left[H_{i j}^{*}\right]$ Matrices defined in equations $\left[a_{i j}\right],\left[b_{i j}\right],\left[d_{i j}\right],\left[h_{i j}\right] \quad \begin{aligned} & (10) \text {. } \\ & \text { Composite compliance submat- }\end{aligned}$

$C_{1}, C_{2}$

$C_{i j}$

$D_{1}$

$E_{L}, E_{T}$ rices defined in equations (9).

Constants defined in equations (20).

Stiffness submatrices for the composite plate; defined in equations (11).

Constants appearing in equation (17).

Cauchy three-dimensional elastic-stiffness coefficients.

Constant appearing in equations (18).

Young's moduli in fibre (L) and transverse (T) directions.

\section{$N_{r}, N_{r \theta}$}

$Q_{1}, Q_{2}$

$$
\begin{aligned}
& f_{1}, f_{2} \\
& G_{L T} \\
& h \\
& i, j
\end{aligned}
$$

$K_{1}, K_{2}$

$M_{i}$

$M_{r}, M_{r \theta}$

$m_{1}, m_{2}$

$N_{i}$

\section{$Q_{i j}$}

$r, \theta$

$u, v, w$

$x, y$

$z$

$\overline{\boldsymbol{\epsilon}}_{\boldsymbol{i}}$

$\kappa_{i}$

$\bar{\nu}$

$\nu_{a}, \nu_{b}, \nu_{d}$

$\nu_{L T}, \nu_{T L}$

$\rho$

$\rho_{0}, \rho_{1}$

$\sigma_{i}$

$\sigma_{L}, \sigma_{T}$

$\tau_{z x}, \tau_{z y}$

$\omega$

()$_{x}$
Body forces, per unit area, in $x$, $y$ directions.

Shear modulus related to $\mathrm{L}, \mathrm{T}$ directions.

Composite-plate thickness.

Indices that take on values 1,2 , 6; 1 and 2 refer to normal-type actions in directions $x, y ; 6$ refers to $x y$ shear action.

Constants defined in equations (20).

Bending moments $(i=1,2)$ and twisting moment $(i=6)$ per unit length.

Radial-bending and twisting moments, per unit length, related to $r, \theta$ co-ordinates.

Body couples, per unit area, in $x, y$ directions.

Membrane forces, per unit length, related to $x y$ co-ordinate system $(i=1,2,6)$.

Radial and shear membrane forces, per unit length, related to plane polar co-ordinate system $r$, $\theta$.

Thickness-shear stress resultants acting on edges cut by $x$ and $y$ co-ordinates.

Plane-stress reduced-stiffness coefficients.

Plane polar co-ordinates in the plane of the plate.

Displacements in $x, y, z$ directions.

Rectangular co-ordinates in the plane of the plate.

Thickness-direction co-ordinate, measured from the midplane of the composite plate.

Midplane engineering-strain components related to $x y$ coordinate system $(i=1,2,6)$.

Curvature-change components related to $x y$ co-ordinate system $(i=1,2,6)$.

Effective isotropic in-plane Poisson's ratio.

Extensional, coupling, and flexural Poisson's ratios for isotropic case.

Major and minor Poisson's ratios related to $\mathrm{L}, \mathrm{T}$ directions.

Material density.

Composite mass coefficients defined in equations (3).

Stress components related to $x y$ co-ordinate system $(i=1,2,6)$. Stresses in fibre and transverse directions.

Thickness shear stresses in the $z x$ and $z y$ planes.

Rotational speed.

$=\partial() / \partial x$. 


\section{ANALYSIS}

\subsection{Basic assumptions}

The analysis presented is based on classical theory for thin, laminated plates, as presented in chapters II-VI of (11). However, it is necessary to add body forces and body moments due to the midplane symmetric and asymmetric parts of the mass distribution within the laminate.

For completeness, the assumptions inherent in the analysis are stated explicity as follows.

(1) The displacements, slopes, and strains are all so small that the classical linear strain-displacement relations are adequate.

(2) The disc is sufficiently thin compared with its radius for the Kirchhoff hypothesis to be used. This implies that the only non-zero strain components are the three in-plane strains, that these vary linearly with the distance from the mid-plane, and that the plate is in a state of plane stress in its plane.

(3) The disc is assumed to consist of a completely arbitrary lamination arrangement, in which each layer is made of homogeneous, rectangular orthotropic, linear elastic material of arbitrary thickness and oriented at any arbitrary angular orientation. The behaviour of a rectangular orthotropic material with its major material-symmetry axis oriented arbitrarily is mechanically equivalent to that of a material which is generally anisotropic with respect to rectangular co-ordinates. It is assumed that the layers are bonded together with a perfect bond.

(4) The disc is assumed to be solid circular, of uniform thickness, and rotating at a uniform speed about its axis. The only generalized forces considered are the quasi-static ones due to the centrifugal action.

(5) In this analysis, the disc is assumed to be free at its edge. However, by means of superposition, other edge conditions could be included without much difficulty.

\subsection{Basic equations}

The $x$ and $y$ axes are rectangular cartesian co-ordinates fixed in the mid-plane of the disc and rotating with it. The stress components in this co-ordinate system are denoted in contracted composite-material notation (chapter 2 of (14)) as follows: $\sigma_{1}$ and $\sigma_{2}$ are normal stresses in the $x$ and $y$ directions and $\sigma_{6}$ is the shear stress acting along the $x$ and $y$ directions (Fig. 3). All of the other stress components are absent, since the disc is assumed to be in a state of plane stress (hypothesis 2 ).

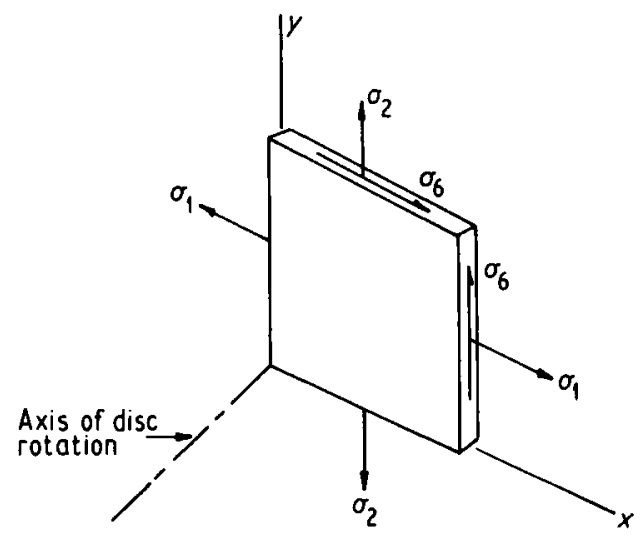

Fig. 3. Element of one layer of the disc showing co-ordinate axes and stress components
The stress resultants and stress couples are defined as follows:

$$
\left(N_{i}, M_{i}\right)=\int_{-h / 2}^{h / 2}(1, z) \sigma_{i} \mathrm{~d} z(i=1,2,6)
$$

where $z$ is the thickness-direction position co-ordinate measured from the mid-plane of the composite disc and $h$ is the total thickness of the composite disc (Fig. 4).

The respective body forces and body moments, per unit plate area, in the $x$ and $y$ directions are as follows (Fig. 4):

$$
\left(f_{1}, f_{2}\right)=\rho_{0} \omega^{2}(x, y) ;\left(m_{1}, m_{2}\right)=\rho_{1} \omega^{2}(x, y) \text {. }
$$

where $\omega$ is the rotational speed,

$$
\left(\rho_{0}, \rho_{1}\right)=\int_{-h / 2}^{h / 2}(1, z) \rho \mathrm{d} z
$$

and $\rho$ is the material density which may vary in stepwise fashion from layer to layer.

The respective in-plane force, moment, and thicknessdirection force-equilibrium equations that must be satisfied are:

$$
\left.\begin{array}{l}
\begin{array}{l}
N_{1, x}+N_{6, y}+f_{1}=0 \\
N_{6, x}+N_{2, y}+f_{2}=0
\end{array} \\
M_{1, x}+M_{6, y}-Q_{1}+m_{1}=0 \\
M_{6, x}+M_{2, y}-Q_{2}+m_{2}=0 \\
Q_{1, x}+Q_{2, y}=0
\end{array}\right\}
$$
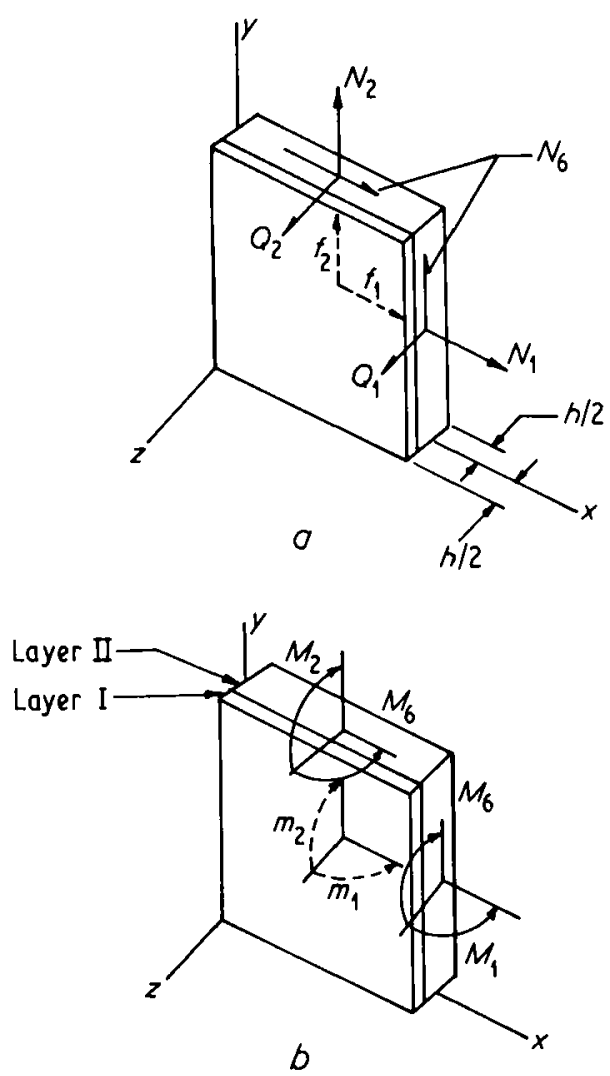

$a$ Stress resultants and body forces. $b$ Stress couples and body couples.

Fig. 4. Schematic diagrams 
where the thickness-shear-stress resultants are defined as

$$
\left(Q_{1}, Q_{2}\right)=\int_{-h / 2}^{h / 2}\left(\tau_{z x}, \tau_{z y}\right) \mathrm{d} z
$$

The rectangular anisotropic constitutive relations (hypothesis 3) are, from chapter 3 of (14),

$\left\{\begin{array}{c}\bar{\epsilon}_{1} \\ \bar{\epsilon}_{2} \\ \bar{\epsilon}_{6} \\ \hdashline \kappa_{1} \\ \kappa_{2} \\ \kappa_{6}\end{array}\right\}=\left[\begin{array}{ccc:ccc}a_{11} & a_{12} & a_{16} & b_{11} & b_{12} & b_{16} \\ a_{12} & a_{22} & a_{26} & b_{12} & b_{22} & b_{26} \\ a_{16} & a_{26} & a_{66} & b_{16} & b_{26} & b_{66} \\ \hdashline h_{11} & h_{12} & h_{16} & d_{11} & d_{12} & d_{16} \\ h_{12} & h_{22} & h_{26} & d_{12} & d_{22} & d_{26} \\ h_{16} & h_{26} & h_{66} & d_{16} & d_{26} & d_{66}\end{array}\right]\left\{\begin{array}{c}N_{1} \\ N_{2} \\ N_{6} \\ \hdashline M_{1} \\ M_{2} \\ M_{6}\end{array}\right\}$

where the composite compliance submatrices $a_{i j}, b_{i j}, d_{i j}, h_{i j}$ are defined as

$$
\text { as } \left.\begin{array}{rl}
{\left[a_{i j}\right]} & =\left[A_{i j}^{*}\right]-\left[B_{i j}^{*}\right]\left[D_{i j}^{*}\right]^{-1}\left[H_{i j}^{*}\right] \\
{\left[b_{i j}\right]} & =\left[B_{i j}^{*}\right]\left[D_{i j}^{*}\right]^{-1} \\
{\left[h_{i j}\right]} & =-\left[D_{i j}^{*}\right]^{-1}\left[H_{i j}^{*}\right],\left[d_{i j}\right]=\left[D_{i j}^{*}\right]^{-1}
\end{array}\right\}
$$

Here

$$
\left.\begin{array}{l}
{\left[A_{i j}^{*}\right]=\left[A_{i j}\right]^{-1},\left[B_{i j}^{*}\right]=-\left[A_{i j}\right]^{-1}\left[B_{i j}\right]} \\
{\left[H_{i j}^{*}\right]=\left[B_{i j}\right]\left[A_{i j}\right]^{-1}} \\
{\left[D_{i j}^{*}\right]=\left[D_{i j}\right]-\left[B_{i j}\right]\left[A_{i j}\right]^{-1}\left[B_{i j}\right]}
\end{array}\right\}
$$

and the respective composite stiffness submatrices are defined as

$$
\left(A_{i j}, B_{i j}, D_{i j}\right) \equiv \int_{-h / 2}^{h / 2}\left(l, z, z^{2}\right) Q_{i j} \mathrm{~d} z(i, j=1,2,6)
$$

where the $Q_{i j}$ are the plane-stress reduced-stiffness coefficients obtained from the Cauchy three-dimensional stiffness coefficients as follows (15):

$$
Q_{i j}=C_{i j}-\left(C_{i 3} C_{j 3} / C_{33}\right)(i, j=1,2,6)
$$

Owing to the symmetry of the general array of $C_{i j}$, we have $Q_{j i}=Q_{i j}$. Equations useful in calculating the $Q_{i j}$ from the more familiar elastic properties, Young's and shear moduli and Poisson's ratio, are given in Appendix 1.

The mid-plane strains $\bar{\epsilon}_{i}$ and curvature changes $\kappa_{i}$ are defined in terms of the displacements $u, v, w$ in the respective $x, y, z$ directions by the following linear relations (hypothesis 1):

$$
\left.\begin{array}{l}
\bar{\epsilon}_{1}=u,_{x} ; \bar{\epsilon}_{2}=v,_{y} ; \bar{\epsilon}_{6}=v,_{x}+u,_{y} \\
\kappa_{1}=-w,_{x x} ; \kappa_{2}=-w,_{y y} ; \kappa_{6}=-2 w,_{x y}
\end{array}\right\} .
$$

Thus, the following strain- and curvature-change compatibility relations must hold (4): $\dagger$

†The derivation of equation (5) is presented in Appendix 2

$$
\begin{aligned}
& \bar{\epsilon}_{1, y y}+\bar{\epsilon}_{2, x x}-\bar{\epsilon}_{6, x y}=0 \\
& \kappa_{1, y y}+\kappa_{2, y y}-\kappa_{6, x y}=0 .
\end{aligned}
$$

The boundary conditions to be satisfied are those associated with a free edge along $r=R$ (hypothesis 5), where $R$ is the radius of the disc.

$$
N_{r}=0, N_{r \theta}=0 ; M_{r}=0, M_{r \theta}=0
$$

as well as the regularity conditions at the centre of the disc, which is assumed to be solid (hypothesis 4), i.e. $N_{1}, N_{2}$, $N_{6}, M_{1}, M_{2}, M_{6}$ must all be finite at the disc axis $(x=y=$ $0)$.

Equations (1)-(16) constitute a complete mathematical formulation of the problem.

\subsection{Closed-form solution}

The problem is formulated in terms of the membrane forces and moments. Any set of functions $N_{1}, N_{2}, N_{6}, M_{1}, M_{2}$ and $M_{6}$ that satisfy equations (1)-(16) constitute the unique solution of the problem.

Motivated by Lekhnitskii's solution for the homogeneous orthotropic case (3) and the solution for the laminated isotropic case (4), one may assume that the membrane forces per unit length are of the following form:

$$
\left.\begin{array}{l}
N_{1}=1 / 2 \omega^{2}\left[C_{1}\left(x^{2}+3 y^{2}-R^{2}\right)\right. \\
\left.+C_{2}\left(R^{2}-x^{2}-y^{2}\right)\right] \\
N_{2}=1 / 2 \omega^{2}\left[C_{1}\left(3 x^{2}+y^{2}-R^{2}\right)\right. \\
\left.+C_{2}\left(R^{2}-x^{2}-y^{2}\right)\right] \\
N_{6}=-1 / 2 \omega^{2}\left(2 C_{1} x y\right)
\end{array}\right\}
$$

where $C_{1}$ and $C_{2}$ are constants to be determined. Equations (17) satisfy the appropriate boundary conditions, the first two of equations (16), as well as the regularity conditions on $N_{1}, N_{2}$, and $N_{6}$. To satisfy the in-plane equilibrium equations (4), it is necessary to set $C_{2}=\rho_{0}$.

In analogous fashion, the moments per unit length are

$$
\left.\begin{array}{l}
M_{1}=1 / 2 \omega^{2}\left[D_{1}\left(x^{2}+3 y^{2}-R^{2}\right)\right. \\
\left.+\rho_{1}\left(R^{2}-x^{2}-y^{2}\right)\right] \\
M_{2}=1 / 2 \omega^{2}\left[D_{1}\left(3 x^{2}+y^{2}-R^{2}\right)\right. \\
\left.+\rho_{1}\left(R^{2}-x^{2}-y^{2}\right)\right] \\
M_{6}=-1 / 2 \omega^{2}\left(2 D_{1} x y\right)
\end{array}\right\} . . . .
$$

where $D_{1}$ is a constant to be determined.

Inserting equations (17), (18), and (8) into equations (14) and (15) one obtains the following results:

$$
\left.\begin{array}{l}
C_{1}=\left(D K_{1}-B K_{2}\right) /(A D-B H) \\
D_{1}=\left(-H K_{1}+A K_{2}\right) /(A D-B H)
\end{array}\right\} \text {. }
$$


where

$$
\begin{aligned}
& A \equiv 3\left(a_{11}+a_{22}\right)+2 a_{12}+a_{66} \\
& B \equiv 3\left(b_{11}+b_{22}\right)+2 b_{12}+b_{66} \\
& D \equiv 3\left(d_{11}+d_{22}\right)+2 d_{12}+d_{66} \\
& H \equiv 3\left(h_{11}+h_{22}\right)+2 h_{12}+h_{66} \\
& K_{1} \equiv\left(a_{11}+2 a_{12}+a_{22}\right) \rho_{0} \\
& +\left(b_{11}+2 b_{12}+b_{22}\right) \rho_{1} \\
& K_{2} \equiv\left(h_{11}+2 h_{12}+h_{22}\right) \rho_{0} \\
& +\left(d_{11}+2 d_{12}+d_{22}\right) \rho_{1}
\end{aligned}
$$

\section{DISCUSSION}

Equations (17) with $C_{2}=\rho_{0}$ and (18) with $C_{1}$ and $D_{1}$ given by equations (19) constitute the closed-form solution of the approximate title problem. For a homogeneous, rectangular orthotropic disc $B=0$ and $\rho_{1}=0$ and all terms with subscripts 16 and 26 vanish. The result coincides with that of Lekhnitskii (3). For a disc arbitrarily laminated of isotropic materials, $a_{11}=a_{22}=a, b_{11}=b_{22}=b, d_{11}=d_{22}=d$, $a_{66}=2\left(1+\nu_{a}\right) a, b_{66}=2\left(1+\nu_{b}\right) b, d_{66}=2\left(1+\nu_{d}\right) d$, where $\nu_{a} \equiv-a_{12} / a_{11}, \nu_{b} \equiv-b_{12} / b_{11}$, and $\nu_{d} \equiv-d_{12} / d_{11}$. Then the present solution coincides with that derived in (4) by an entirely different formulation and method of solution, but using the same compatibility equations.

\section{NUMERICAL EXAMPLES}

\subsection{Example 1: Glass-epoxy/steel}

The numerical example is selected to illustrate the importance of the bending-stretching coupling effect, as well as the orthotropic effect which occurs even when the stiffnesses $Q_{11}$ and $Q_{22}$ associated with the respective $x$ and $y$ directions are equal. The laminate is chosen to be a two-layer one to exaggerate the bending-stretching coupling effect. Layer I is steel and layer II is fibreglass-epoxy, assumed to have the material properties listed in Table 1.

The maximum normal stresses (bending plus membrane) always occur at the centre $(r=0)$ of a solid disc. The maximum normal stresses occurring in the two layers are depicted graphically in dimensionless form as a function of the thickness ratio (thickness of steel divided by total thickness) in Fig. 5 (solid curves).

It is seen that the thickness ratio has a strong influence on the maximum stresses. The dimensionless maximum stress in the steel has its minimum value at a thickness ratio of unity, and the dimensionless maximum stress in the glass-epoxy is also minimized at the same thickness ratio. Fig. 5 shows that the steel curve is especially sensitive at

Table 1. Layer-material parameters for example 1

\begin{tabular}{l|c|c}
\hline \multicolumn{1}{c|}{ Parameter } & $\begin{array}{c}\text { Material I } \\
\text { (steel) }\end{array}$ & $\begin{array}{c}\text { Material II } \\
\text { (fibreglass-epoxy)* }\end{array}$ \\
\hline Young's modulus E, $\mathrm{lb} / \mathrm{in}^{2}$ & $29.5 \times 10^{6}$ & $3.26 \times 10^{6}$ \\
Poisson's ratio $\nu$ & 0.28 & 0.12 \\
Shear modulus, $\mathrm{lb} / \mathrm{in}^{2}$ & $=\mathrm{E} /[2(1+\nu)]$ & $0.810 \times 10^{6}$ \\
Specific weight, $\mathrm{lb} / \mathrm{in}^{3}$ & 0.283 & 0.071 \\
\hline
\end{tabular}

*181-style fabric (16).

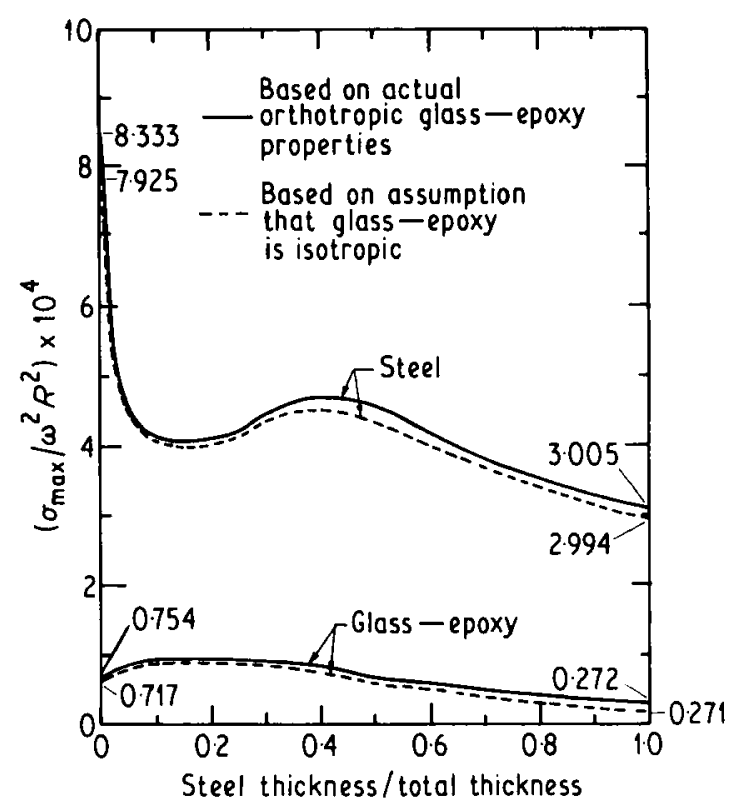

Fig. 5. Effect of thickness ratio on the maximum dimensionless normal stresses in the individual layers of a twolayer rotating disc

small values of the thickness ratio: decreasing the steel thickness in this range causes a dramatic increase in stress in the steel. In contrast, both curves are especially insensitive at large values of the thickness ratio: changes in glassepoxy thickness for a disc that is largely made of steel has little effect on the stresses.

The question arises of the effect of the material orthotropy of the glass-epoxy on the calculated maximum stress, since this material has a very special kind of orthotropy in that $E_{L}=E_{T}=E$ and thus $\nu_{L T}=\nu_{T L}=\nu$, but $G$ $\neq E /[2(1+\nu)]$. The results of calculations for this case are depicted by the dashed curves in Fig. 5 . It is clear that, for this particular combination of materials, the effect of material orthotropy of one layer is not nearly as pronounced as the effect of asymmetric lamination.

This example illustrates the obvious disadvantage of asymmetrically laminating a multi-material rotating disc. Sometimes, however, it may not be possible to avoid asymmetrical lamination. An example may be a wooden block mounted on a steel faceplate for turning on a lathe. The wooden block may be laminated plywood or may even be monolithic, since wood is itself an orthotropic material owing to its cellular structure as manifested in its 'grain'.

\subsection{Example 2: Two-layer, cross-ply graphite-epoxy}

This example is selected to emphasize the effect of a highly orthotropic material $\left(E_{L} / E_{T} \gg 1\right)$ utilized in the most simple lamination scheme (two-layer cross-ply; see Fig. $2 e$ ).

The properties used are taken from (17) and are listed in Table 2. The effect of varying the cross-ply ratio (ratio of the $0^{\circ}$-layer thickness to the total thickness) is shown in Fig. 6. It is not surprising that use of a cross-ply ratio of 0.5 (i.e. $0^{\circ}$ and $90^{\circ}$ layers having the same thickness) results in an increase in the maximum normal stress reached in either layer compared with that reached in a single-layer disc. In the two-layer cross-ply case, bending-stretching coupling is always induced even when the cross-ply ratio is 0.5 . However, it is to be emphasized that this does not mean that the 
Table 2. Individual-layer material properties for composite materials in Examples 2 and 3

\begin{tabular}{l|l|l}
\hline \multicolumn{1}{c|}{ Property } & $\begin{array}{c}\text { Example 2 } \\
(\text { Graphite- } \\
\text { epoxy*) }\end{array}$ & $\begin{array}{c}\text { Example 3 } \\
\text { (Organic fibre- } \\
\text { epoxy**) }\end{array}$ \\
\hline Major Young's modulus $E_{L}, \mathrm{lb} / \mathrm{in}^{2}$ & $25.0 \times 10^{6}$ & $11.0 \times 10^{6}$ \\
Minor Young's modulus $E_{T}, \mathrm{lb} / \mathrm{in}^{2}$ & $1.13 \times 10^{6}$ & $0.8 \times 10^{6}$ \\
Major Poisson's ratio $\nu_{L T}$ & 0.35 & 0.34 \\
Shear modulus $\left(G_{L T}\right), \mathrm{lb} / \mathrm{in}^{2}$ & $0.77 \times 10^{6}$ & $0.3 \times 10^{6}$ \\
Specific weight $(\rho g), \mathrm{lb} / \mathrm{in}^{2}$ & 0.055 & 0.050 \\
\hline
\end{tabular}

*Thornel-50 fibres-4617 epoxy resin (17).

**Kevlar 49 fibres (0.60 volume fraction) - epoxy resin (18).

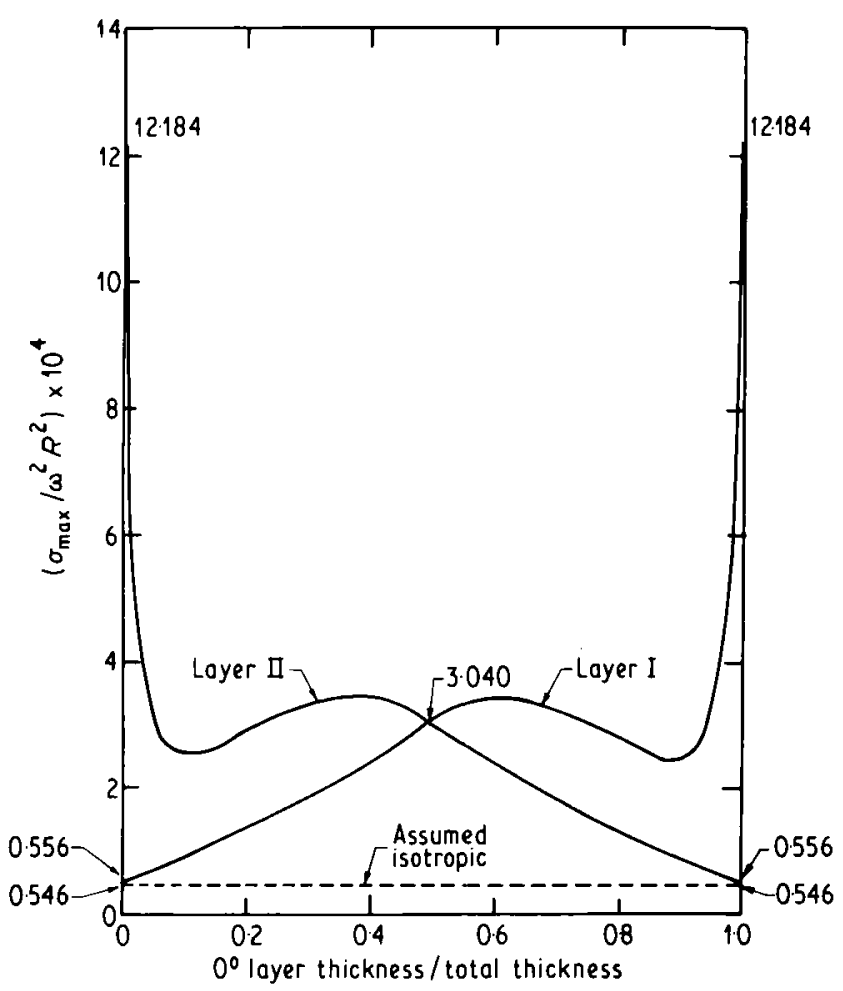

Fig. 6. Effect of cross-ply ratio on the maximum dimensionless stresses in the individual layers of a two-layer, cross-ply graphite-epoxy rotating disc

single-layer disc is the best graphite-epoxy design to use, since in the cross-ply design the transverse normal stresses are much less than in the single-layer design, for which transverse normal stress would undoubtedly be the governing failure criterion. The present paper is limited to stress analysis only and the topics of multiaxial-stress failure criterion and optimal design are not considered.

The dashed curve shown in Fig. 6 is obtained by making the approximation of an 'equivalent' isotropic disc having $E$ $=\left(E_{L} \mathrm{E}_{T}\right)^{1 / 2}$ and $\nu=\left(\nu_{L T} \nu_{T L}\right)^{1 / 2}$. It is seen that this approximation gives a very inaccurate and exaggerated prediction of maximum stress.

4.3 Example 3: Quasi-isotropic layups of organic fibreepoxy

The quasi-isotropic lamination scheme, originated by Werren and Norris (6), consists of orthotropic layers oriented in such a way that the resulting elastic stretching stiffness (the $\left[A_{i j}\right]$ submatrix) is isotropic, i.e. it has stiffness coefficients that are independent of orientation in the plane. The necessary and sufficient conditions that must be met to achieve such a result are (8):

the total number of layers $(n)$ must be 3 or more;

the individual layers (denoted by index $k$, ranging from 1 to $n$ ) must have identical orthotropic elastic coefficients $Q_{i j}$ and layer thicknesses;

all interfacial bonds must be perfect, i.e. there must be no interlayer shear deformation, slip, or debonding;

each layer in a set of layers (denoted by index $K$ ) must be oriented at an angle $\theta_{K}=\pi(K-1) / S$ with respect to some reference direction, where $S$ is the number of such sets.

Since a laminate made according to the above design is isotropic in regard to its stretching submatrix $\left[A_{i j}\right]$ only and not, in general, in regard to its bending-stretching coupling and bending stiffnesses, $\left[B_{i j}\right]$ and $\left[D_{i j}\right]$, this design is called quasi-isotropic or pseudo-isotropic.

The simplest lamination scheme meeting the criteria listed above is the three-layer lay-up consisting of one layer at $0^{\circ}$, one at $60^{\circ}$, and another at $120^{\circ}$ (Fig. 2a). There are three combinations of such arrangements:

$0^{\circ} / 60^{\circ} / 120^{\circ}$, suggested by Rabenhorst et al. (9)

$0^{\circ} / 120^{\circ} / 60^{\circ}$

$60^{\circ} / 0^{\circ} / 120^{\circ}$

The numerical results for the dimensionless maximum normal stresses for these three kinds of lamination scheme are presented in Table 3, which is based on properties listed in the last column of Table 2. It appears that the $0^{\circ} / 60^{\circ} / 120^{\circ}$ and $0^{\circ} / 120^{\circ} / 60^{\circ}$ lamination schemes are equally better than the $60^{\circ} / 0^{\circ} / 120^{\circ}$ arrangement. It is interesting that the first two schemes had identical maximum stresses in the layers. Thus, so far as maximum stresses are concerned, interchanging the $60^{\circ}$ and $120^{\circ}$ layers had no effect for this kind of loading. The explanation for this is that the individual-layer orientation angles $\theta_{k}$ appear only as even powers of $\sin \theta_{k}$ and $\cos \theta_{k}$ in the stress expressions.

The next to the simplest quasi-isotropic lamination scheme is the four-layer one consisting of one layer each at $0^{\circ}, 45^{\circ}, 90^{\circ}$, and $135^{\circ}$. So far as the effects on the stresses are concerned, the $45^{\circ}$ and $135^{\circ}$ layers can be interchanged (just as the $60^{\circ}$ and $120^{\circ}$ layers could be interchanged in the three-layer case), but the $0^{\circ}$ and $90^{\circ}$ layers cannot be interchanged. Then there are six distinct lamination arrangements; they are listed, along with the dimensionless

Table 3. Dimensionless maximum normal stress in each

layer for various three-layer quasi-isotropic lay-ups of organic fibre-epoxy composite material

\begin{tabular}{c|c|c|c|c}
\hline $\begin{array}{c}\text { Lamination } \\
\text { scheme }\end{array}$ & Layer & $\begin{array}{c}\text { Orientation, } \\
\text { degrees }\end{array}$ & $\begin{array}{c}\left(\sigma_{L \max } / \omega^{2} R^{2}\right) \\
\times 10^{4}\end{array}$ & $\begin{array}{c}\left(\sigma_{T \max } / \omega^{2} R^{2}\right) \\
\times 10^{4}\end{array}$ \\
\hline \multirow{3}{*}{$3 \mathrm{~A}$} & 1 & 0 & 1.746 & 0.349 \\
& 2 & 60 & 2.511 & 0.255 \\
& 3 & 120 & 1.396 & 0.308 \\
\hline \multirow{3}{*}{$3 \mathrm{~B}$} & 1 & 0 & 1.746 & 0.349 \\
& 2 & 120 & 2.511 & 0.255 \\
& 3 & 60 & 1.396 & 0.308 \\
\hline $3 \mathrm{C}$ & 1 & 60 & 2.840 & 0.185 \\
& 2 & 0 & 1.070 & 0.272 \\
& 3 & 120 & 2.840 & 0.185 \\
\hline
\end{tabular}


maximum normal stresses, in Table 4, which is based on the properties listed in the last column of Table 2. It appears that lamination scheme $4 \mathrm{~F}$ is best since it has the smallest value of maximum normal stress. It is interesting that this lamination scheme has a maximum stress that is approximately 7.8 per cent lower then the best three-layer quasiisotropic arrangement (3A or 3B).

It is noted that in the best three-ply ( $3 \mathrm{~A}$ and $3 \mathrm{~B}$ ) and four-ply (4F) quasi-isotropic lamination arrangements, the stress distributions are not symmetrical about the mid-plane of the plate. This suggests that detrimental bendingstretching coupling action is taking place. This action can be eliminated by appropriate combination of two $3 \mathrm{~A}$ or two $4 \mathrm{~F}$ laminates into a single symmetrically laminated sixor eight-layer 'double' laminate. The resulting layer stresses for such lamination schemes $(6 \mathrm{~A}, 6 \mathrm{~B}$, and $8 \mathrm{~A})$ are listed in Table 5. It is interesting that the stresses are uniform throughout the thickness and that all three of these arrangements $(6 \mathrm{~A}, 6 \mathrm{~B}$, and $8 \mathrm{~A})$ have the same maximum stresses (approximately 60.8 per cent smaller stress than $3 \mathrm{~A}$ or 57.5 per cent smaller than $4 F$ ).

In the patent specification of Rabenhorst et al. (9) the following seven-ply lamination scheme is shown: $120^{\circ} / 60^{\circ} / 0^{\circ} / 120^{\circ} / 0^{\circ} / 60^{\circ} / 120^{\circ}$. Although this lamination arrangement is symmetric about the laminate mid-plane, it is not quasi-isotropic and this results in a higher maximum stress than do the double quasi-isotropic lay-ups, see Table 5.

For the $6 \mathrm{~A}, 6 \mathrm{~B}$, and $8 \mathrm{~A}$ lay-ups, the facts that the maximum normal stresses in the directions along the fibres $(\mathrm{L})$ and transverse to the fibres (T) are uniform through the thickness and that the quasi-isotropic lamination scheme results in the same in-plane stiffnesses in all directions in the plane might suggest that the maximum normal stress

Table 4. Dimensionless maximum normal stress in each layer for various four-layer quasi-isotropic lay-ups of organic fibre-epoxy composite material

\begin{tabular}{|c|c|c|c|c|}
\hline $\begin{array}{l}\text { Lamination } \\
\text { scheme }\end{array}$ & Layer & $\begin{array}{c}\text { Orientation, } \\
\text { degrees }\end{array}$ & $\begin{array}{c}\left(\sigma_{L \max } / \omega^{2} R^{2}\right) \\
\times 10^{4}\end{array}$ & ${ }^{\left(\sigma_{T \max } / \omega^{2} R^{2}\right)}$ \\
\hline $4 A$ & $\begin{array}{l}1 \\
2 \\
3 \\
4\end{array}$ & $\begin{array}{r}0 \\
45 \\
90 \\
135 \\
\end{array}$ & $\begin{array}{l}1.340 \\
2.231 \\
1.644 \\
2.396\end{array}$ & $\begin{array}{l}0.305 \\
0.212 \\
0.318 \\
0.228\end{array}$ \\
\hline $4 B$ & $\begin{array}{l}1 \\
2 \\
3 \\
4 \\
\end{array}$ & $\begin{array}{r}0 \\
90 \\
135 \\
45 \\
\end{array}$ & $\begin{array}{l}0.124 \\
0.078 \\
0.876 \\
2.341 \\
\end{array}$ & $\begin{array}{l}0.075 \\
0.165 \\
0.178 \\
0.222 \\
\end{array}$ \\
\hline $4 C$ & $\begin{array}{l}1 \\
2 \\
3 \\
4\end{array}$ & $\begin{array}{r}0 \\
135 \\
45 \\
90 \\
\end{array}$ & $\begin{array}{l}0.963 \\
2.567 \\
2.043 \\
2.529 \\
\end{array}$ & $\begin{array}{l}0.457 \\
0.244 \\
0.194 \\
0.268 \\
\end{array}$ \\
\hline $4 D$ & $\begin{array}{l}1 \\
2 \\
3 \\
4 \\
\end{array}$ & $\begin{array}{r}45 \\
0 \\
90 \\
135 \\
\end{array}$ & $\begin{array}{l}1.717 \\
1.981 \\
2.062 \\
2.630 \\
\end{array}$ & $\begin{array}{l}0.163 \\
0.221 \\
0.275 \\
0.250 \\
\end{array}$ \\
\hline $4 E$ & $\begin{array}{l}1 \\
2 \\
3 \\
4\end{array}$ & $\begin{array}{r}45 \\
135 \\
0 \\
90 \\
\end{array}$ & $\begin{array}{l}0.944 \\
1.410 \\
1.469 \\
2.500 \\
\end{array}$ & $\begin{array}{l}0.045 \\
0.134 \\
0.198 \\
0.215\end{array}$ \\
\hline $4 \mathrm{~F}$ & $\begin{array}{l}1 \\
2 \\
3 \\
4\end{array}$ & $\begin{array}{r}45 \\
0 \\
135 \\
90\end{array}$ & $\begin{array}{l}2.150 \\
1.616 \\
2.314 \\
1.549\end{array}$ & $\begin{array}{l}0.204 \\
0.303 \\
0.220 \\
0.333\end{array}$ \\
\hline
\end{tabular}

Table 5. Dimensionless maximum normal stress in each layer of selected six-, seven-, and eight-layer laminates*

\begin{tabular}{c|c|c|c|c}
\hline $\begin{array}{c}\text { Lamination } \\
\text { scheme }\end{array}$ & Layer & $\begin{array}{c}\text { Orientation, } \\
\text { degrees }\end{array}$ & $\begin{array}{c}\left(\sigma_{L \max } / \omega^{2} R^{2}\right) \\
\times 10^{4}\end{array}$ & $\begin{array}{c}\left(\sigma_{T \max } / \omega^{2} R^{2}\right) \\
\times 10^{4}\end{array}$ \\
\hline
\end{tabular}

Double three-layer, symmetrically laminated, quasi-isotropic lay-ups

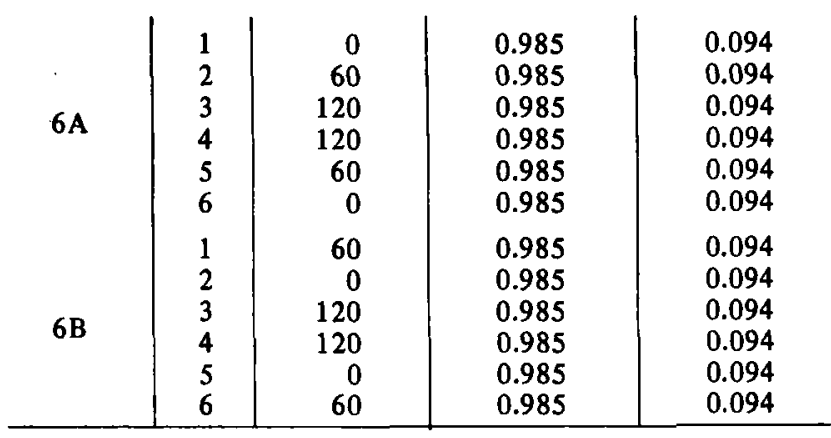

Seven-layer, symmetrically laminated lay-ups suggested in (9)

\begin{tabular}{l|l|r|l|l} 
& & & & \\
7 & 120 & 0.959 & 0.098 \\
7 & 2 & 60 & 0.959 & 0.098 \\
& 3 & 0 & 1.110 & 0.091 \\
& 4 & 120 & 0.959 & 0.098 \\
& 5 & 0 & 1.110 & 0.091 \\
& 6 & 60 & 0.959 & 0.098 \\
& 7 & 120 & 0.959 & 0.098 \\
\hline
\end{tabular}

Double four-layer, symmetrically laminated quasi-isotropic lay-up

\begin{tabular}{l|r|r|l|l} 
& 1 & 0 & 0.985 & 0.094 \\
$8 \mathrm{~A}$ & 2 & 45 & 0.985 & 0.094 \\
& 3 & 90 & 0.985 & 0.094 \\
& 4 & 135 & 0.985 & 0.094 \\
& 5 & 135 & 0.985 & 0.094 \\
& 6 & 90 & 0.985 & 0.094 \\
& 7 & 45 & 0.985 & 0.094 \\
& 8 & 0 & 0.985 & 0.094 \\
\hline
\end{tabular}

*Material: organic fibre-epoxy with properties given in Table 2.

could be calculated from the following simple expression applicable to a homogeneous isotropic disc, provided that the effective isotropic in-plane Poisson's ratio $\bar{D}$ is calculated from Werrens and Norris' equation (see Appendix 1):

$$
\sigma_{\max } / \omega^{2} R^{2}=(3+\nu)(\rho / 8) \text {. . . . . . . . }
$$

Equation (21) yields a value of $\sigma_{\max }=0.540 \times 10^{-4}$. However, it of course predicts that $\sigma_{\max }$ is equal to this value in all directions, i.e. the sum of the dimensionless principal stresses is $1.080 \times 10^{-4}$. In lamination arrangements $6 \mathrm{~A}, 6 \mathrm{~B}$, and $8 \mathrm{~A}$, the sum of the dimensionless principal stresses is essentially identical, namely $1.079 \times 10^{-4}$. Thus the principal-stress sum is invariant, but there is no simple way to determine a priori the separate values of $\sigma_{L_{\max }}$ and $\sigma_{T_{\max }}$.

\section{ACKNOWLEDGEMENTS}

The author wishes to thank Mark Bohon, senior in mechanical engineering, for suggesting this problem. He is also grateful to Professor Eric Reissner of the University of California at San Diego for pointing out the approximate nature of equation (15), as discussed in Appendix 2. The programming assistance of Timothy Chen, mechanicalengineering graduate student, and the use of the computational facilities of the Merrick Computing Center are also acknowledged. 


\section{APPENDIX 1}

\section{SOME COMPOSITE-MATERIAL RELATIONS}

For a single, thin layer of orthotropic composite material, let $E_{L}$ and $E_{T}$ denote major and minor Young's moduli, $\nu_{L T}$ the major Poisson's ratio and $G_{L T}$ the shear modulus related to material-symmetry axes. The minor Poisson's ratio $v_{T L}$ can be determined from the reciprocal relation:

$$
\nu_{T L}=\nu_{L T}\left(E_{T} / E_{L}\right) \text {. }
$$

The plane-stress reduced stiffness coefficients related to the material-symmetry axes $(\mathrm{L}$ and $\mathrm{T})$ are:

$$
\left.\begin{array}{l}
Q_{L L}=E_{L} / \lambda ; Q_{L T}=\nu_{L T} E_{T} / \lambda \\
Q_{T T}=E_{T} / \lambda ; Q_{S S}=G_{L T}
\end{array}\right\} .
$$

where

$$
\lambda \equiv 1-\nu_{L T} \nu_{T L}
$$

To transform the properties from the material-symmetry directions $(\mathrm{L}, \mathrm{T})$ of an individual layer to arbitrary orthogonal reference directions $(1,2)$, the following transformation equations may be used:

$\left\{\begin{array}{l}Q_{11} \\ Q_{12} \\ Q_{22} \\ Q_{16} \\ Q_{26} \\ Q_{66}\end{array}\right\}=\left[\begin{array}{llll}m^{4} & 2 m^{2} n^{2} & n^{4} & 4 m^{2} n^{2} \\ m^{2} n^{2} & m^{4}+n^{4} & m^{2} n^{2} & -4 m^{2} n^{2} \\ n^{4} & 2 m^{2} n^{2} & m^{4} & 4 m^{2} n^{2} \\ -m^{3} n & m^{3} n-m n^{3} & m n^{3} & \left(m^{2}-n^{2}\right)^{2} \\ -m n^{3} & -m^{3} n+m n^{3} & m^{3} n & 2 m^{3} n-2 m n^{3} \\ m^{2} n^{2} & -2 m^{2} n^{2} & m^{2} n^{2} & -2 m^{3} n+2 m n^{3}\end{array}\right\}$

$$
\left\{\begin{array}{l}
Q_{L L} \\
Q_{L T} \\
Q_{T T} \\
Q_{S S}
\end{array}\right\}
$$

Assuming that the layer properties are macroscopically homogeneous through the thickness of each individual layer, one can rewrite equations (11) as follows:

$$
\left.\begin{array}{l}
A_{i j}=\sum_{k=1}^{\mathrm{n}}\left(z_{k}-z_{k-1}\right) Q_{i j}{ }^{(k)} \\
B_{i j}=\frac{1}{2} \sum_{k=1}^{\mathrm{n}}\left(z_{k}^{2}-z_{k-1}^{2}\right) Q_{i j}{ }^{(k)} \\
D_{i j}=\frac{1}{3} \sum_{k=1}^{\mathrm{n}}\left(z_{k}^{3}-z_{k-1}^{3}\right) Q_{i j}^{(k)}
\end{array}\right\}
$$

The effective in-plane Poisson's ratio of a quasi-isotropic laminate can be expressed as follows (6):

$$
\begin{aligned}
& \bar{\nu}=\left(E_{L}+E_{T}+6 \nu_{L T} E_{T}-4 \lambda G_{L T}\right) /\left(3 E_{L}+3 E_{T}\right. \\
& \left.+2 \nu_{L T} E_{T}+4 \lambda G_{L T}\right) \text {. . . . . . . . . }
\end{aligned}
$$

\section{APPENDIX 2}

\section{DISCUSSION OF THE CURVATURE COMPATIBILITY CONDITION}

The curvature changes are related to the displacements by the following linear relations:

$$
\kappa_{1}=-w_{, x x} ; \quad \kappa_{2}=-w_{, y y} ; \quad \kappa_{6}=-2 w,_{x y} . . .
$$

Integrating the first two of equations $(B-1)$, one obtains

$$
w_{x_{x}}=-\int \kappa_{1} d x+f(y) ; \quad w_{, y}=-\int \kappa_{2} d y+g(x)
$$

Differentiating equations (27) and (28) appropriately, we get

$$
\begin{array}{ll}
w_{x_{x y}}=-\frac{1}{2} \kappa_{6, x} ; & w_{x_{x x y}}=-\kappa_{1, y} \\
w_{x y y}=-\frac{1}{2} \kappa_{6, y} ; & w_{x_{x y y}}=-\kappa_{2, x}
\end{array}
$$

Thus, the equations necessary and sufficient to ensure compatibility of the three curvature-change components are as follows:

$$
\kappa_{1, y}-\frac{1}{2} \kappa_{6, x}=0 ; \quad \kappa_{2, x}-\frac{1}{2} \kappa_{6, y}=0 .
$$

The two first-order equations (30) are not analogous to the single second-order equation (14) governing midplane strain compatibility. To obtain a single second-order equation in the curvature components directly analogous to equation (14), one may differentiate the first of equations (30) with respect to $y$ and the second with respect to $x$ and then add the resulting two equations to obtain equation (15).

Since all solution of equations (30) necessarily satisfy equation (15), the latter equation is a necessary condition for curvature compatibility. However, it is noted that solutions of equation (15) must satisfy the following equations:

$$
\kappa_{1, y}-\frac{1}{2} \kappa_{6, x}=\bar{f}(x) ; \quad \kappa_{2, x}-\frac{1}{2} \kappa_{6, y}=\bar{g}(y) .
$$

where $\bar{f}(x)$ and $\bar{g}(y)$ are arbitrary functions of $x$ and $y$, respectively. It is clear that all solutions of equations (31), and thus of equation (15), do not satisfy equations (30). Therefore, equation (15) is not a sufficient condition for curvature compatibility and in using it in this paper, we are obtaining only approximate solutions.

\section{APPENDIX 3}

\section{REFERENCES}

(1) TIMOSHENKO, S. P. History of strength of materials 1953 270 (McGraw-Hill Book Co. Inc., New York and London).

(2) LEKHNITSKII, S. G. Anisotropic plates 2nd edition (English translation) 1968151 (Gordon and Breach, New York)

(3) ibid. 146

(4) BERT, C. W. 'A new formulation for the theory of thin elastic plates', 1974 (unpublished).

(5) SHAW, M. C. 'Wheels for higher-speed grinding', Mech. Engng 197193 (8), 19-23.

(6) WERREN, F, and NORRIS, C. B. 'Mechanical properties of laminate designed to be isotropic', 1953 Report No. 1841 (Forest Products Laboratory, Madison, Wisc.).

(7) TSAI, S. W. and PAGANO, N. J. 'Invariant properties of composite materials', Composite materials workshop (edited by $\mathrm{S}$. W. Tsai. J. C. Halpin, and N. J. Pagano) 1968 (Technomic Publishing Co., Westport, Conn.).

(8) BERT, C. W. 'Analysis of plates', Chapter 4 in Structural analysis and design, Pt I (edited by C. C. Chamis), Vol. 7 in Composite materials (edited by L. J. Broutman and R. H. Krock) 1975 (Academic Press, New York). 
(9) RABENHORST, D. W., BRANDT, A., DARNELL, K. E., and GEORGE, J. F. 'Pseudo-isotropic filament disc structures' U.S. Patent 3788162 (Jan. 29, 1974).

(10) WHITNEY, J. M. 'Bending-extensional coupling in laminated plates under transverse loading', J. compos. mater., 19693 , $20-28$.

(11) ASHTON, J. E. and WHITNEY, J. M. Theory of laminated plates 1970 (Technomic Publishing Co., Westport, Conn.)

(12) DEB, D. 'The stresses - in a transversely isotropic rotating circular plate with rigid core', J. Aerospace Sci. 1962 29, $1003-1004$.

(13) WU, N. and RAMSEY, J. H. 'Stresses in a layered rotating disc', Int. J. Mechanical Sciences 1966 8, 629-639.

(14) ASHTON, J. E., HALPIN, J. C., and PETIT, P. H. Primer on composite materials: analysis 1969 (Technomic Publishing Co., Westport, Conn.)

(15) TSAI, S. W. 'Mechanics of composite materials, Pt II: Theoretical aspects', 1966 Report AFML-TR-66-149, Pt II (Air Force Materials Laboratory, Wright-Patterson Air Force Base, Ohio).

(16) ANON 'Plastics for tlight vehicles. Pt I: Reinforced plastics', MIL-HDBK-17, 1959 (Armed Forces Supply Support Center, Washington, D.C.).

(17) DONER, D. R. and NOVAK, R. C. 'Structural behaviour of laminated graphite filament composites', 24 th Annual Tech. Conf. 1969 (Reinforced Plastics/Composites Division, Society of the Plastics Industry, Washington, D.C., Section 2-D).

(18) Kevlar 49 data manual 1974 (E. I. du Pont de Nemours \& Co. Inc., Textile Fibres Dept, Wilmington, Del.). 